\title{
Day-Ahead Corrective Adjustment of FACTS Reactance: A Linear Programming Approach
}

\author{
M. Sahraei-Ardakani, Member, IEEE, and K. W. Hedman, Member, IEEE
}

\begin{abstract}
Reserve requirements serve as a proxy for $\mathrm{N}-1$ reliability in the security-constrained unit commitment (SCUC) problem. However, there is no guarantee that the reserve is deliverable for all scenarios (post-contingency states). One cheap way to improve reserve deliverability is to harness the flexibility of the transmission network. Flexible AC transmission system (FACTS) devices are able to significantly improve the transfer capability. However, FACTS utilization is limited today due to the complexities these devices introduce to the DC optimal power flow problem (DCOPF). With a linear objective, the traditional DCOPF is a linear program (LP); when variable impedance based FACTS devices are taken into consideration, the problem becomes a nonlinear program (NLP). A reformulation of the NLP to a mixed integer linear program, for day-ahead corrective operation of FACTS devices, is presented in this paper. Engineering insight is then introduced to further reduce the complexity to an LP. Although optimality is not guaranteed, the simulation studies on the IEEE 118-bus system show that the method finds the globally optimal solution in $98.8 \%$ of the cases. Even when the method did not find the optimal solution, it was able to converge to a nearoptimal solution, which substantially improved the reliability, very quickly.
\end{abstract}

Index Terms - FACTS devices, linear programming, power system reliability, power transmission reliability, transfer capability, transmission topology optimization.

\section{NOMENCLATURE}

Sets

G Set of generators.

$g \quad$ Index of generators, $g \in G$.

$K \quad$ Set of all transmission elements, line or transformer.

$k \quad$ Index of transmission lines, $k \in K$.

$\bar{K} \quad$ Set of transmission lines equipped with FACTS, $\bar{K} \subset K$.

$\bar{k} \quad$ Index of lines equipped with FACTS, $\bar{k} \in \bar{K} \subset K$.

$\underline{K} \quad$ Set of transmission elements not equipped with FACTS, $\underline{K} \subset K$.

$\underline{k} \quad$ Index of transmission elements not equipped with FACTS, $\underline{k} \in \underline{K} \subset K$.

$N \quad$ Set of nodes.

$n \quad$ Index for nodes, $n \in N$.

$T \quad$ Set of time periods.

$t \quad$ Index for time periods, $t \in T$.

$\delta^{+}(n)$ Set of lines specified as to node $n$.

This manuscript was submitted on January 23, 2015.

Mostafa Sahraei-Ardakani and Kory W. Hedman are with the School of Electrical, Computer, and Energy Engineering, Arizona State University, Tempe, AZ 85287 USA (e-mail: Mostafa@asu.edu; Kory.Hedman@asu.edu). $\delta^{-}(n)$ Set of lines specified as from node $n$.

$g(n)$ Set of generators connected to node $n$.

$n(g)$ Node location of generator $g$.

\section{Parameters}

$B_{k} \quad$ Electrical susceptance of line $k$.

$B_{\bar{k}}^{\max }$ Maximum susceptance of line $\bar{k}$ equipped with FACTS.

$B_{\bar{k}}^{\min } \quad$ Minimum susceptance of line $\bar{k}$ equipped with FACTS.

$c_{g} \quad$ Operation cost of unit $g(\$ / M W h)$.

$c_{g}^{N L} \quad$ No-load cost of unit $g$.

$c_{g}^{S D} \quad$ Shut-down cost of unit $g$.

$c_{g}^{S U} \quad$ Start-up cost of unit $g$.

$P_{g}^{\max }$ Maximum output of unit $g$.

$P_{g}^{\min }$ Minimum output of unit $g$.

$F_{k}^{\max }$ Capacity of transmission line $k$.

$F_{k}^{\max , c}$ Emergency rate c of transmission line $k$.

$R_{g}^{H R} \quad$ Maximum hourly ramp rate of unit $g$.

$R_{g}^{10} \quad$ Maximum 10-min ramp rate of unit $g$.

$U T_{g} \quad$ Minimum up time of unit $g$.

$D T_{g} \quad$ Minimum down time of unit $g$.

$u_{g t}^{*} \quad$ Scheduled unit commitment status of generation unit $g$ in period $t$.

$P_{g t}^{*} \quad$ Scheduled real power output of unit $g$ in period $t$.

$F_{\bar{k}}^{*} \quad$ Scheduled real power flow on line $\bar{k}$ in period $t$.

$R S V_{t}^{R e q}$ Required level of spinning reserve in period $t$.

\section{Variables}

$u_{g t} \quad$ Unit commitment binary variable for generation unit $g$ in period $t$.

$v_{g t} \quad$ Start-up binary variable for generation unit $g$ in period $t$.

$w_{g t} \quad$ Shut-down binary variable for generation unit $g$ in period $t$.

$P_{g t} \quad$ Real power output variable for unit $g$ in period $t$.

$R S V_{g t}$ Scheduled spinning reserve for unit $g$ in period $t$.

$F_{k t} \quad$ Real power flow through line $k$ in period $t$.

$d_{n t} \quad$ Demand at bus $n$ in period $t$.

$\theta_{n t} \quad$ Voltage angle at node $n$ in period $t$.

$d_{n t}^{-} \quad$ Load not served at node $n$ in period $t$.

$d_{n t}^{+} \quad$ Over generation at node $n$ in period $t$.

$B_{\bar{k}} \quad$ Susceptance of line $\bar{k}$ equipped with FACTS.

$z_{\bar{k}} \quad$ Binary variable indicating the sign of voltage angle difference on line $\bar{k}$ equipped with FACTS. 


\section{INTRODUCTION}

$\mathrm{E}^{\mathrm{x}}$ XPLICIT modeling of N-1 reliability criterion in securityconstrained unit commitment (SCUC) adds a great deal of complexity to the problem. Existing state of the art optimization solvers struggle to handle such large-scale stochastic programming problems within the available time. Some lead modern SCUC solvers may include explicit representations of a very limited subset of the reliability constraints. Therefore, reserve requirements are used as a proxy for the reliability needs in existing SCUC solvers. However, there is no guarantee that the reserve is deliverable in all possible scenarios (e.g., post-contingency states). Thus, system operators make necessary adjustments to the day-ahead market solution to ensure reliability. Such adjustments are made out-of-market (referred to as out-of-market corrections, OMC) [1] and may include a variety of actions, such as committing additional units, redispatching units away from the market solution, and disqualifying reserve that is selected by the approximate market model. OMC actions are not preferred as they are costly and they distort price signals. Therefore, there is a great interest in software (modeling and algorithms) advances in order to reduce operator adjustments to the market solution.

One way to improve reserve deliverability is by harnessing the flexibility of the transmission grid [2]. Transmission switching (TS) has been shown to enhance the transfer capability leading to economic [3]-[7] and reliability benefits [8]. TS can also be used as a corrective mechanism to reduce postcontingency violations [9]-[10]. Continuous adjustment of lines' reactance can also achieve the same goals with less stability concerns [11]-[18]. One technology that would allow such control is flexible AC transmission system (FACTS) devices [19]-[20]. It is estimated that FACTS devices can improve the transfer capability over the existing grid by a factor of fifty percent [21]. However, like transmission switching [22], optimal adjustment of FACTS devices introduces nonconvexities to the DC optimal power flow (DCOPF), which makes it extremely challenging to solve within a reasonable time with existing computational power [23]. This paper contributes to the literature by developing a fast algorithm that provides suggestions to the operator on potential corrective FACTS setting adjustments for the day-ahead solution. The operator can implement the corrective adjustment in the realtime after the relevant contingency. A similar formulation has been proposed for economic improvement in [24], where FACTS adjustments are included in security-constrained economic dispatch (SCED) formulations. The method developed in [24] is a two-stage LP that would suggest FACTS adjustments aiming at enhancement of transfer capability, leading to a cheaper generation dispatch. This paper proposes the inclusion of FACTS adjustments in SCUC as a corrective mechanism, to enhance reserve deliverability. Therefore, this paper focuses on reliability benefits of corrective FACTS adjustments in contingency analysis, while [24] aims at achieving economic benefits. To fully harness the transmission network flexibility, development of a fast algorithm for corrective adjustment of FACTS set point is of great interest.

Different types of FACTS for a variety of applications exist today [25]. In this paper, we loosely use the term "FACTS" for the specific types of FACTS devices that are able to significantly affect the reactance of a line and, hence, provide nota- ble power flow control. Unified power flow controllers (UPFC) and thyristor controlled series compensators (TCSC) are examples for FACTS devices that provide such control by significantly adjusting the reactance of transmission lines. The adjustment can be either only inductive or both capacitive and inductive, depending on the device. The formulation provided in this paper can also be used for unconventional FACTS technology such as the variable reactance devices designed under Advanced Research Projects Agency - Energy (ARPAE) Green Electricity Network Integration (GENI) initiative, including the Smart Wire Grid device [26]. These unconventional FACTS devices can only provide inductive adjustments.

Previous research shows that FACTS devices help the system in many ways. A Benders' decomposition based formulation is developed in [27], for inclusion of FACTS in day-ahead SCUC to reduce the cost and improve the reliability. The tradeoff between system cost and risk is studied in the presence of corrective power control provide by FACTS devices in [28]. Reference [29] shows that social welfare gains can be achieved as a result of having demand response and FACTS in the system at the same time. Such body of literature clearly and correctly points to the different benefits of incorporating FACTS devices in various stages of power system operation. However, there still exist essential problems that need to be addressed.

Major challenges that prevent better utilization of the transmission network through operation of FACTS devices are the following: (i) cost of these devices; (ii) legacy operational philosophies that prefer minimal changes to the existing system; (3) computational complexity of incorporating FACTS devices in OPF. It should be noted, however, that even the FACTS devices that are already installed are not being utilized to their full extent. This underutilization is one source of inefficiency that exists in the power system as pointed out by existing literature. Previous research has discussed this problem and proposed different methods, such as transmission bidding [30] and compensation based on market value [31]-[33], to operate FACTS devices more efficiently. The underutilization of existing FACTS devices are due to legacy EMS and MMS systems, and also the computational complexity of an OPF that is capable of incorporating these devices. This computational challenge has not been properly addressed yet. For instance, [34]-[36] propose various methods to include FACTS adjustment for a variety of applications. However, the formulation developed in [34]-[37] is computationally expensive and will not be applicable to large-scale power systems. A regression-based model is proposed in [38] to provide suggestions to the operator regarding the operation of FACTS devices and improve the usage of transmission capacity; this prior work aims at addressing the computational complexity challenge by employing an offline statistical method. While [38] aims at a key challenge, the statistical method they used has natural limitations. For instance, the model heavily depends on the offline simulation of potential states and would only be useful if the system operates at a state close to the simulated conditions. An iterative sensitivity-based LP approach is developed in [39], which suggests corrective FACTS adjustments to relieve post-contingency overloads. The method in [39] is relatively fast, but it is sensitivity-based and iterative, and thus may not scale well. Moreover, the formulation developed in 
[39] does not include the full OPF model, e.g., generation redispatch, when determining the FACTS settings. A formal formulation of the problem and a method based on optimization theory seems to be more convincing and comprehensible. Moreover, optimization based formulation of the problem can provide better insight into the potential solutions. This paper uses formal operations research to develop an algorithm that is fast and produces reliable solutions.

The mathematical representation of a DC optimal power flow (DCOPF) while considering the flexibility of FACTS devices is a nonlinear program (NLP). Solving such a problem, within the available timeframe, is beyond existing computational capabilities. This paper contributes to the literature by reformulating the NLP to a linear program (LP), which can be solved quickly. The formulation is focused on the dayahead corrective adjustment of FACTS devices to enhance the deliverability of the reserves. First, the NLP is reformulated to a mixed integer linear program (MILP). The integer variables in the MILP represent the sign of voltage angle differences on the transmission lines with the corresponding FACTS devices. These signs dictate the direction of power flow on the corresponding transmission lines. By enforcing the power flow to be in the same direction for the lines equipped with FACTS, the MILP can be solved only for the node representing the precontingency flow direction, i.e., it is possible to solve an LP in this case instead of an MILP. Such an approach reduces the complexity of the problem from an MILP to an LP. The solution to the resulting LP may or may not be optimal depending on the direction of the power flows in the globally optimal solution coming from the original MILP. Note that it is extremely unlikely that the direction of power flow on the lines equipped with FACTS change due to corrective adjustment of the FACTS devices' set point. Often times, FACTS adjustment reroutes the power flow on the parallel paths to the uncongested lines, which would involve adjustment of power flow on the same direction. This engineering insight is confirmed with $98.8 \%$ success rate in 2760 simulation studies conducted in section IV. Even if the optimal solution involves reversing the flow on at least one of the lines equipped with FACTS, the significance of this work does not disappear. It suggests corrective switching adjustments very quickly, which will improve the system reliability, although the adjustments may not be guaranteed to be optimal.

\section{FORMULATION}

A standard SCUC is presented in (1)-(14). ISOs run some form of a SCUC to clear the day-ahead market (DAM) and determine the hourly status and schedule of the units a day prior to operation.

$$
\begin{array}{lr}
\operatorname{Min} \sum_{t} \sum_{g} c_{g} P_{g t}+c_{g}^{N L} u_{g t}+c_{g}^{S U} v_{g t}+c_{g}^{S D} w_{g t} & \forall g, t \\
P_{g t} \geq P_{g}^{\text {min }} u_{g t} & \forall g, t \\
P_{g t}+R S V_{g t} \leq P_{g}^{\text {max }} u_{g t} & \forall g, t \\
\sum_{s=t-U T_{g}-1}^{t} v_{g s} \leq u_{g t} & \forall g, t \\
\sum_{s=t-D T_{g}-1}^{t} w_{g s} \leq 1-u_{g t} & \forall g, t \\
v_{g t}-w_{g t}=u_{g t}-u_{g t-1} & \forall k, t \\
P_{k t}-B_{k}\left(\theta_{n t}-\theta_{m t}\right)=0 & \forall k, t \\
-F_{k}^{\max } \leq F_{k t} \leq F_{k}^{\text {max }} & \forall n, t \\
\sum_{k \in \delta^{+}(n)} F_{k t}-\sum_{k \in \delta^{-}(n)} F_{k t}+\sum_{g \in g(n)} P_{g t}=d_{n t} & \forall n, t
\end{array}
$$

$$
\begin{array}{ll}
R_{g}^{H R} u_{g, t-1}+P_{g}^{\max } v_{g t} \geq P_{g t}-P_{g t-1} & \forall g, t \\
R_{g}^{H R} u_{g t}+P_{g}^{\text {max }} w_{g t} \geq P_{g t-1}-P_{g t} & \forall g, t \\
R S V_{t}^{\text {Req }} \leq \sum_{g} R S V_{g t} & \forall t \\
R S V_{g t} \leq R_{g}^{10} u_{g t} & \forall g, t \\
u_{g t} \in\{0,1\} ; 0 \leq v_{g t}, w_{g t} \leq 1 & \forall g, t
\end{array}
$$

While the SCUC formulation incorporates some form of reliability constraints, the solution to SCUC is not necessarily $\mathrm{N}-1$ reliable. In existing SCUCs, proxy reserve requirements are used instead of solving a stochastic SCUC where N-1 reliability constraints are explicitly included in the formulation (such a formulation is referred to as an extensive form stochastic program and can be solved directly or by a decomposition approach). Some modern SCUC implementations include explicit modeling of a very limited subset of $\mathrm{N}-1$ reliability constraints as well as limited feedback from the contingency analysis package. Even for such lead modern SCUC solvers, proxy reserve requirements are the main reliability instrument, and, thus, reserved deliverability is not guaranteed. Therefore, the ISO has to check whether the system is able to withstand the critical contingencies. ISOs check for a subset of the contingencies, while the approach presented in this paper checks for all the potential N-1 (generator and transmission) events. These practices vary from system to system, because each system is unique and the level of complexity is different. If the solution was not N-1 reliable, the ISO would implement OMC actions, e.g., turn on additional units, to ensure reliability. OMC actions are expensive, inefficient (increase system costs), and also distort price signals. Thus, they are not preferred by system operators. The additional flexibility that FACTS devices introduce to the system can be used to improve the deliverability of the reserves and reduce the need for OMC actions. FACTS set-point adjustment is a viable corrective solution that provides desirable power flow control as a response to the occurrence of a particular contingency when the reserve is not deliverable. Fig. 1 shows the structure of a day-ahead market when FACTS adjustment is included in the contingency analysis tool. This additional flexibility would improve the deliverability of the reserve and reduce the potential post-contingency violations. Therefore, the need for OMC actions would be reduced.

If the contingency analysis tool identifies that a contingency would result in network violations, further steps are necessary. Particularly, (15)-(24) present an optimization problem with the objective of minimizing the violations. Network violations consist of load shedding and over-generation due to the inability of the system to provide proper transfer capability for the reserves. Load shedding occurs when there is not enough transfer capability to deliver the reserve to the desirable location. Similarly, when the transfer capability and ramping limits do not allow the energy produced by a generator to be transferred to demand, over generation will happen. This standard formulation would allow the generators to adjust their output within their ramping limits to serve the load in the post-contingency state. FACTS flexibility is introduced to the problem in (20)-(21), providing power flow control to enhance the deliverability of the reserves. Equations (20)-(21) state that the lines equipped with FACTS devices have variable susceptance and their power flow will be affected by this variable susceptance. This additional flexibility is presented by an ad- 
ditional variable and its corresponding value in the solution would represent the optimal corrective adjustments needed for a particular contingency.

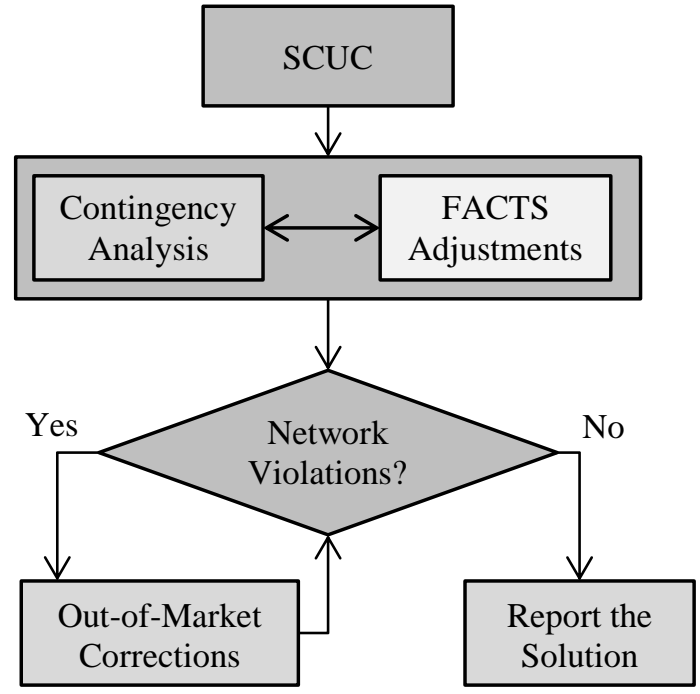

Fig. 1. Day-ahead market including FACTS adjustment inside the contingency analysis tool.

In the case of a generation contingency, the output of that particular generator is set to zero; in the case of a transmission contingency, the flow on that particular line is set to zero. The problem allows the generators to change their output within their 10-minute ramping limits, i.e., this formulation assumes that 10-minute operating reserve is used to respond to the contingency. The load shedding and over generation can only be positive. The flows on the lines are constrained to their emergency capacity limits. Note that the problem should be solved for each contingency in each hour.

$\min \sum_{n}\left(d_{n t}^{-}+d_{n t}^{+}\right)$

$P_{g}^{\min } u_{g t}^{*} \leq P_{g t} \leq P_{g}^{\max } u_{g t}^{*}$

$-R_{g}^{10} \leq P_{g t}-P_{g t}^{*} \leq R_{g}^{10}$

$-F_{k}^{\max , c} \leq F_{k t} \leq F_{k}^{\max , c}$

$F_{\underline{k} t}-B_{\underline{k}}\left(\theta_{n t}-\theta_{m t}\right)=0$

$F_{\bar{k} t}-B_{\bar{k} t}\left(\theta_{n t}-\theta_{m t}\right)=0$

$B_{\bar{k}}^{\min } \leq B_{\bar{k} t} \leq B_{\bar{k}}^{\max }$

$\sum_{k \in \sigma^{+}(n)} F_{k t}-\sum_{k \in \sigma^{-}(n)} F_{k t}+\sum_{g \in g(n)} P_{g t}=$

$$
d_{n t}-d_{n t}^{-}+d_{n t}^{+}
$$

$0 \leq d_{n t}^{-} \leq d_{n t}$

$0 \leq d_{n t}^{+}$

It should be noted that (20) is a non-linear constraint since it has the multiplication of $B_{\bar{k}}$ and $\theta$, making (15)-(24) an NLP. However, the constraint can be rewritten as two linear constraints depending on the sign of the voltage angle differences on the line equipped with FACTS. The sign of voltage angle difference identifies the direction of the power flow. For inductive lines, positive voltage angle difference translates to a negative power flow and vice versa. It is the exact opposite for capacitive lines: positive voltage angle difference dictates a positive power flow and vice versa. Equations (25)-(26) show the reformulation of (20)-(21) to linear constraints based on the sign of voltage angle difference.

if $\left(\theta_{n t}-\theta_{m t}\right) \geq 0$ :

$$
\begin{array}{ll}
B_{\bar{k}}^{\min }\left(\theta_{n t}-\theta_{m t}\right) \leq F_{\bar{k} t} \leq B_{\bar{k}}^{\max }\left(\theta_{n t}-\theta_{m t}\right) & \forall \bar{k} \\
\text { if }\left(\theta_{n t}-\theta_{m t}\right) \leq 0: & \\
B_{\bar{k}}^{\max }\left(\theta_{n t}-\theta_{m t}\right) \leq F_{\bar{k} t} \leq B_{\bar{k}}^{\min }\left(\theta_{n t}-\theta_{m t}\right) & \forall \bar{k}
\end{array}
$$

For a positive angle difference $\left(\theta_{n t}-\theta_{m t}\right)$, negative flow on inductive lines, the lower and higher limits on susceptance would also impose the lower and higher limits on the power flow. The exact opposite is true for the case where the voltage angle difference sign is negative. Fig. 2 shows the feasible set identified by (25) and (26). It clearly shows that inclusion of FACTS devices in the DCOPF problem makes the feasible set nonconvex.

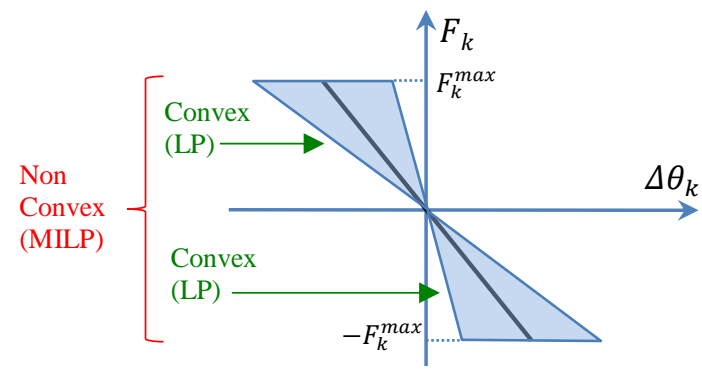

Fig. 2. The feasible set of power flow on a transmission element equipped with FACTS: The solid line shows the flow without consideration of FACTS' impact: $F_{k}=B_{k}\left(\Delta \theta_{k}\right)$, where $\Delta \theta_{k}=\theta_{k, t o}-\theta_{k, \text { from }}$. The shaded area shows the feasible region for power flow on the transmission element considering the impact of FACTS device: $B_{k}^{\min } \leq B_{k} \leq B_{k}^{\max }$, which clearly is a nonconvex set.

The "if conditions" in (25)-(26) can be modeled with binary variables, to create a mixed-integer nonlinear program (MINLP):

$$
\begin{array}{ll}
\left(\left(1-z_{\bar{k} t}\right) B_{\bar{k}}^{\min }+z_{\bar{k} t} B_{\bar{k}}^{\max }\right)\left(\theta_{n t}-\theta_{m t}\right) \geq F_{\bar{k} t} & \forall \bar{k} \\
\left(\left(1-z_{\bar{k} t}\right) B_{\bar{k}}^{\max }+z_{\bar{k} t} B_{\bar{k}}^{\min }\right)\left(\theta_{n t}-\theta_{m t}\right) \leq F_{\bar{k} t} & \forall \bar{k} \\
\left(1-z_{\bar{k} t}\right) \theta_{m t}+z_{\bar{k} t} \theta_{n t} \geq\left(1-z_{\bar{k} t}\right) \theta_{n}+z_{\bar{k} t} \theta_{m t} & \forall \bar{k} \\
z_{\bar{k} t} \in\{0,1\} &
\end{array}
$$

The binary variable, $z_{\bar{k}}$, takes on a value of 1 when the voltage angle difference is positive. A negative voltage angle difference is being represented by $z_{\bar{k}}$ taking on a value of 0 . The problem then can be reformulated to a MILP, using a big $M$ (a big multiplier) reformulation technique:

$$
\begin{array}{ll}
z_{\bar{k} t} B_{\bar{k}}^{\min }\left(\theta_{n t}-\theta_{m t}\right)-\left(1-z_{\bar{k} t}\right) M \leq F_{\bar{k} t} & \forall \bar{k} \\
\left(1-z_{\bar{k} t}\right) B_{\bar{k}}^{\max }\left(\theta_{n t}-\theta_{m t}\right)-z_{\bar{k} t} M \leq F_{\bar{k} t} & \forall \bar{k} \\
z_{\bar{k} t} B_{\bar{k}}^{\max }\left(\theta_{n t}-\theta_{m t}\right)+\left(1-z_{\bar{k} t}\right) M \geq F_{\bar{k} t} & \forall \bar{k} \\
\left(1-z_{\bar{k} t}\right) B_{\bar{k}}^{\min }\left(\theta_{n t}-\theta_{m t}\right)+z_{\bar{k} t} M \geq F_{\bar{k} t} & \forall \bar{k} \\
\theta_{n t}+\left(1-z_{\bar{k} t}\right) M \geq \theta_{m t} & \forall \bar{k} \\
\theta_{m t}+z_{\bar{k} t} M \geq \theta_{n t} & \forall \bar{k} \\
z_{\bar{k} t} \in\{0,1\} & \\
M \gg \operatorname{Max}\left\{F_{\bar{k} t}+B_{k t}\left(\theta_{m t}-\theta_{n t}\right)\right\} &
\end{array}
$$

The problem represented by (15)-(19), (22)-(24), (31)-(38) is a MILP that minimizes the post-contingency violations by improving the reserve deliverability via optimal operation of FACTS devices. This problem is solved for each contingency in each hour and, thus, the solution would represent corrective adjustments only for that particular contingency in that specif- 
ic hour. MILPs, just like NLPs, are computationally expensive problems that may not be solvable within the available timeframe. However, the reformulation of the NLP to MILP provides valuable insight into the problem. If the optimal value of the binary variables, $z_{\bar{k}}$, were known, the complexity of the problem would reduce to a LP. Note that $z_{\bar{k}}$ identifies the direction of the power flow on the lines equipped with FACTS devices. For inductive lines, $z_{\bar{k}}$ being 1 imposes a negative power flow, while $z_{\bar{k}}$ being 0 enforces a positive power flow on that line. The exact opposite is true for capacitive lines.

There are many lines for which the operator knows the direction of the flow, e.g., major tie lines. Even if the direction is not clear, this problem is being solved as a part of the contingency analysis tool. Therefore, the SCUC solution is already available and the pre-contingency direction of the power flows is known. When the SCUC solution fails to withstand a particular contingency, it is due to the transmission system not being able to deliver the reserve to the desirable location. In such situations, it is appropriate to redirect the power flows on the paths parallel to the congested lines. The desirable flow control gained by FACTS should support this logic of sending more power on the same direction to the paths parallel to the congested lines. Thus, it is highly unlikely that the direction of the power flow on lines, which are equipped with FACTS, will change in direction relative to the solution of the SCUC. The simulation studies presented in Section IV confirm this engineering judgment with a very high success rate. Using this engineering judgment, the binary variables can be fixed to correspond to the solution of the SCUC that represents the pre-contingency state, which again assumes that the power flow direction does not change. For inductive lines, $z_{\bar{k}} t$ is set to 0 for the case of positive power flow and 1 for negative power flow. The exact opposite is true for capacitive lines.

To summarize, the following procedure describes how a contingency analysis tool with an LP-based method for corrective adjustment of FACTS set point would function:

1. The SCUC solution is sent to the contingency analysis package.

2. For each hour, the contingency analysis tool checks whether the system withstands the potential contingencies. In this paper, we simulate all possible N-1 events excluding the failure of radial lines. However, system operators often have a pre-identified list of critical contingencies that they take into the consideration and ignore the other potential events. Note that this approach is amenable to any chosen set of contingencies, including multiple contingencies.

3 . If the tool identifies a contingency with network violations, the optimization problem (15)-(19), (22)-(24), (31)-(38) is set up. This problem is an MILP.

4. Using the pre-contingency power flows on the lines equipped with FACTS $\left(F_{\bar{k} t}^{*}\right)$ and the instructions provided above, variables $z_{\bar{k} t}$ are fixed to create an LP.

5. The LP is solved and corrective FACTS adjustments are stored for potential real-time implementation. If the violations are not completely eliminated, the potential violations are reported to the system operator for further necessary OMC actions.

This LP-based method would very quickly give the opera- tor suggestions regarding corrective adjustment of the FACTS set points; while we are looking at this application from the day-ahead perspective, this algorithm is also practical for realtime applications. Even if the optimal solution is not achieved by this fast approximation method, the merit of the LP-based method does not vanish; this algorithm determines FACTS adjustments very quickly that improve the reliability of the solution, which is very beneficial even if the optimal adjustment is not guaranteed.

\section{SimULATION STUDIES}

The algorithm developed in section III is tested on the IEEE 118 bus system. The data is taken from [40] modified according to [41] with further modifications to match [9]-[10]. The full dataset can be downloaded from [42]. To examine the effectiveness of the method, a SCUC solution is first obtained for the system. The solution is then passed to two contingency analysis tools: one with FACTS adjustment and one without. The potential violations are calculated for both contingency analysis tools to identify the impact of FACTS adjustments on violation reductions. The FACTS adjustment part is simulated both using the LP heuristic method and the full MILP. This enables us to comment on the optimality of the results as well as the computational time benefits of the LP method. Fig 3 . shows the simulation studies procedure.

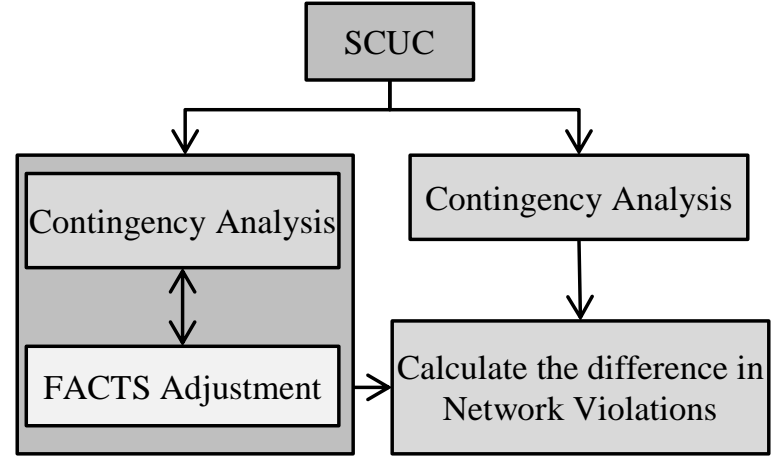

Fig. 3. Simulation studies procedure.

Note that the approach presented in this paper is consistent with the current practices of the industry. PJM has posted a list of switching solutions in response to occurrence of contingencies, consisting of mostly transmission line outages and transmission line flow violations [43]. [44] presents a detailed study of corrective switching on PJM, ERCOT, and TVA. This paper takes a similar approach and uses the flexibility of FACTS devices as a corrective mechanism, instead of transmission line switching. All transmission lines in the IEEE 118 bus system are inductive. The reactance control range tends to be below the line's reactance to avoid issues such as lightly damped oscillations and, thus, all the lines remain inductive even after FACTS adjustments. It is assumed that the FACTS devices can affect the reactance of a line in both inductive and capacitive directions.

The SCUC solution that is obtained withstands all the generator contingencies. However, there are 23 transmission contingencies during the 24 hours that the system is vulnerable to. These contingencies would result in a total $568.76 \mathrm{MW}$ of violations. In order to achieve a good understanding of the performance of the proposed method, several scenarios re- 
garding the sizing, location, and number of FACTS devices are simulated. Fig. 4 shows the violation reductions as a percentage of the total violations for the case where FACTS devices are installed on the lines that are more heavily utilized. Fig. 5 shows the computational time required by the two formulations to solve the problem. Note that the information presented in Fig. 4 and 5 is calculated for the entire day, i.e., the violation reduction and computational time represent all 23 critical contingencies and do not show individual contingency outcomes. Fig. 4 shows that the LP based algorithm developed in this paper is able to find the same solution as the MILP in all cases. The figure also shows that significant violation reductions can be achieved by just a few medium sized FACTS devices if installed on the lines that are more heavily utilized. For instance, 5 FACTS devices with reactance control range of $50 \%$ can eliminate $86 \%$ of the violations and 10 devices with the same control range will make the SCUC solution $100 \%$ reliable. Fig 5 . shows that the LP formulation is solved significantly faster than the original MILP problem.

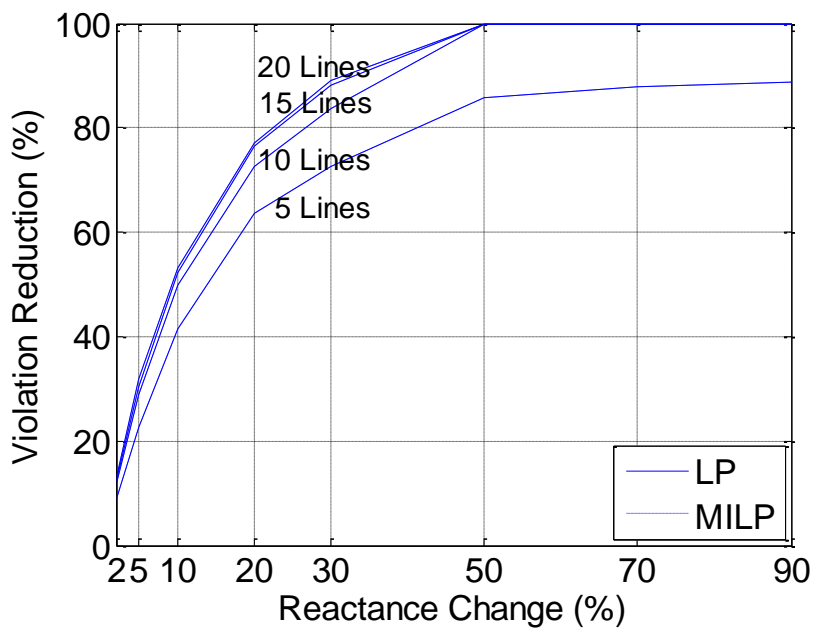

Fig. 4. Violation reduction for LP and MILP FACTS adjustments when FACTS devices are installed on the lines that are more heavily utilized.

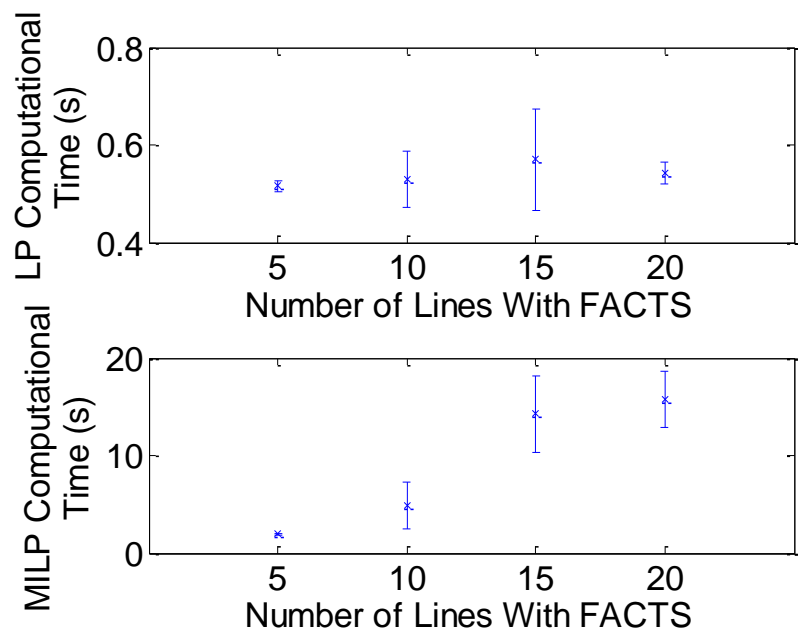

Fig. 5. Computational time for LP and MILP FACTS adjustments when FACTS devices are installed on the lines that are more heavily utilized.

Fig. 6 presents the violation reduction for the case that FACTS devices are installed on the lines with larger reactance. The computational time, shown in Fig. 7, is again much smaller for the LP-based algorithm compared to MILP. A comparison of the LP and MILP violation reductions show that there are some cases for which MILP was able to find a better solution, meaning that our LP-based algorithm converged to a sub-optimal solution; note, however, that the difference in solution quality is minor.

Fig. 8 shows the violation reductions while Fig. 9 presents the computational time for the situation that the FACTS devices are installed on the lines that have lower reactances. The results again confirm that LP-based algorithm is much faster and achieves results that are close to the MILP based method. However, there are cases for which the LP-based algorithm converges to a sub-optimal solution.

Fig. 10 and 11 presents the same information for the case that FACTS devices are installed on the lines with larger capacities. The figures show that the LP-based method converges to the same solution as the MILP based algorithm. However, our LP-based algorithm is much faster than the original MILP problem.

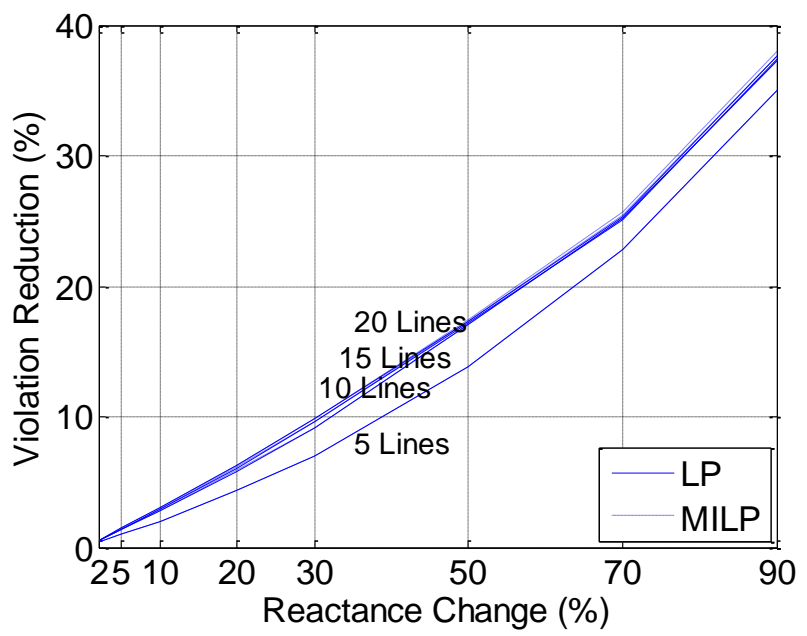

Fig. 6. Violation reduction for LP and MILP FACTS adjustments when FACTS devices are installed on the lines that have larger reactances.

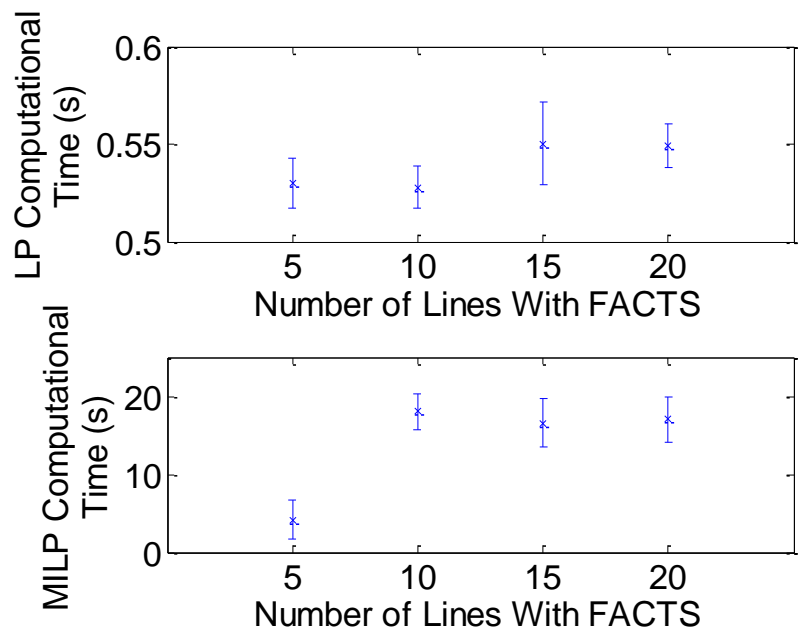

Fig. 7. Computational time for LP and MILP FACTS adjustments when FACTS devices are installed on the lines that have larger reactances. 


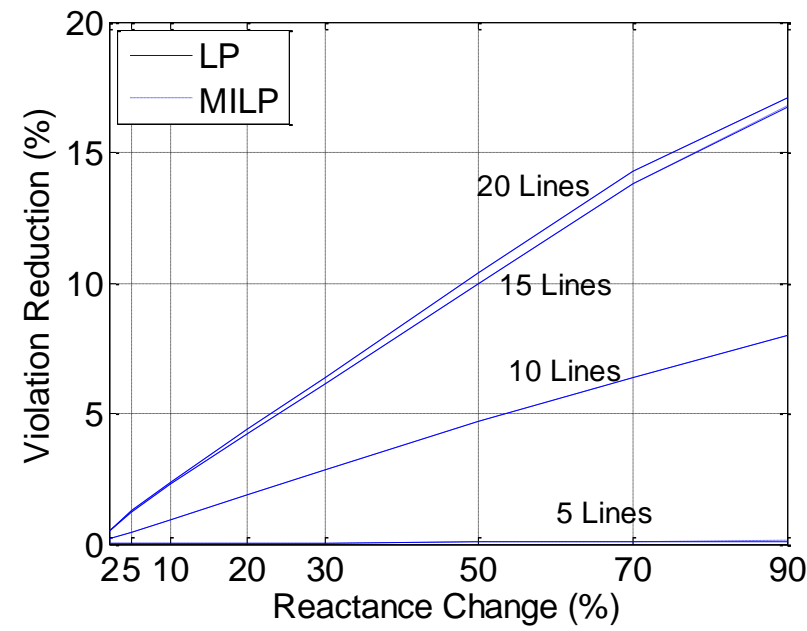

Fig. 8. Violation reduction for LP and MILP FACTS adjustments when FACTS devices are installed on the lines that have smaller reactances.

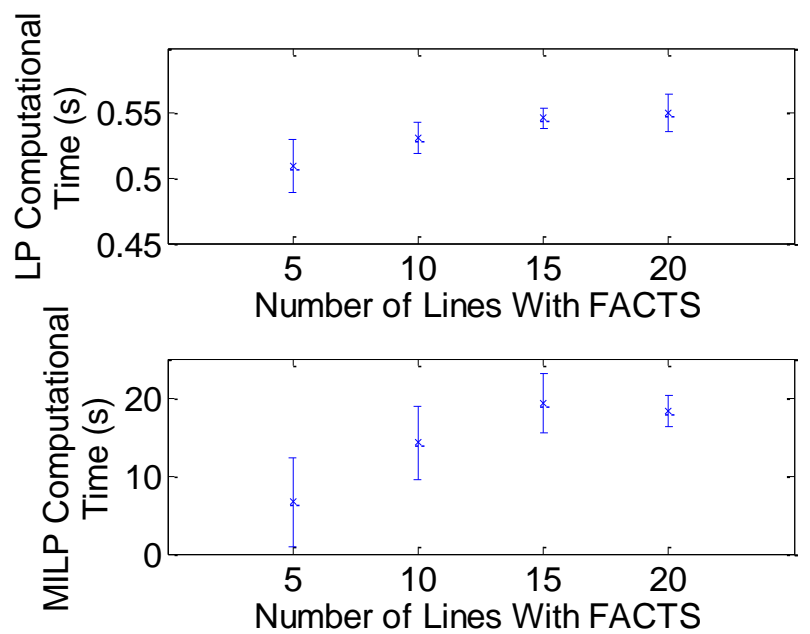

Fig. 9. Computational time for LP and MILP FACTS adjustments when FACTS devices are installed on the lines that have smaller reactances.

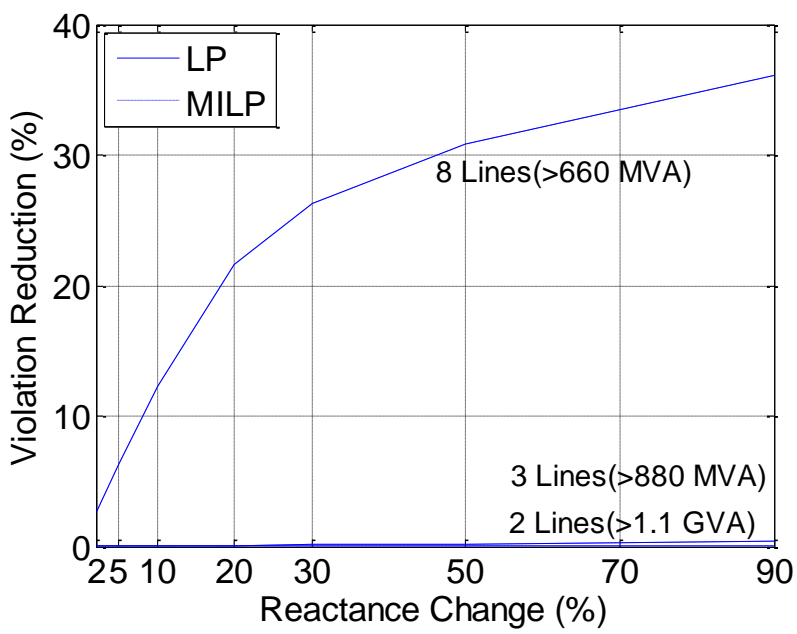

Fig. 10. Violation reduction for LP and MILP FACTS adjustments when FACTS devices are installed on the lines that have larger capacities.

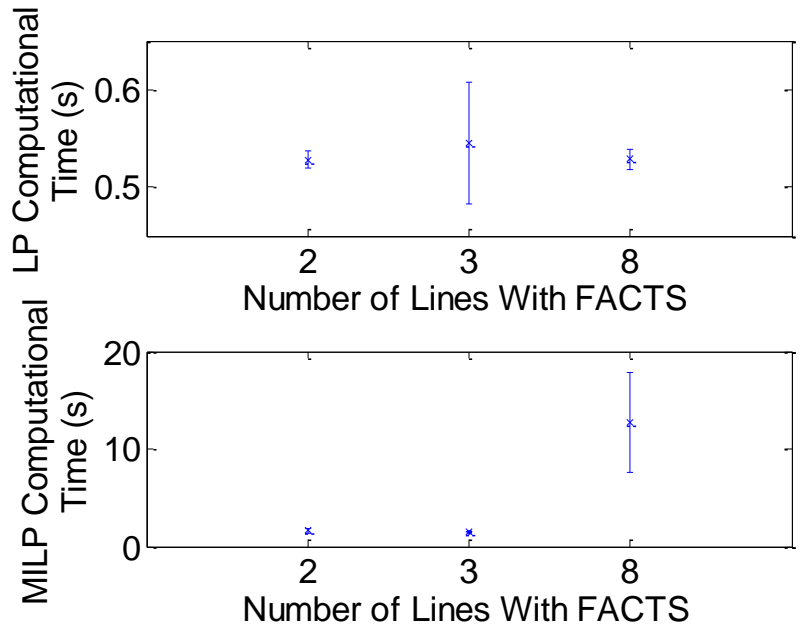

Fig. 11. Computational time for LP and MILP FACTS adjustments when FACTS devices are installed on the lines that have larger capacities.

The results presented in this section have a few implications:

1. Placement of FACTS devices on the lines that are more heavily utilized seems to result in the largest reliability improvements. Previous research shows that the same placement strategy would also lead to sizeable economic gains [24]. This result is consistent with the intuition behind power flow control rationale that the flows should be rerouted around congested lines to alternative paths. Intuitively, placing FACTS devices on lines that are more heavily utilized (congested or operated closer to their capacity) seems to be able to better support such logic. Particularly, increasing the reactance of the congested lines would be an apparent beneficial adjustment. Note, however, that placing FACTS devices on lines that are not as much utilized can also be beneficial but it is harder to identify these beneficial cases. Even if a line is not fully utilized, increasing its impedance will divert the flow to alternative paths but it may also decrease the flow on lines that are downstream from that line, which may be congested. There are more sophisticated FACTS siting methods in the literature. However, to avoid complexities of such allocation algorithms, simple heuristics were used in this paper. Moreover, it was anticipated that the algorithm developed in this paper would perform worse, when FACTS devices were not placed in the right locations, because such siting policies would increase the chances of having the post-adjustment flows in the opposite direction. In fact, this was confirmed by the results presented in this section: the best placement strategy, placement of FACTS on the lines that are more heavily utilized, produced optimal results for all the cases. The suboptimal solutions occurred only under other placement strategies that were not as effective. The method was deliberately simulated on these not so ideal placement strategies, to test the method under these more rigorous conditions. The results show that even under such circumstances, the reformulation developed in the paper would perform well and produce optimal solution (the same solution as MILP) most of the time. The results also enable commenting on the quality of the solutions in cases that the method con- 
verged to suboptimal solution: the suboptimal solutions were very close to the globally optimal solutions.

2. The results included 2760 simulations of individual contingencies. For all but 33 of these simulations ( $1.2 \%$ of all simulations), the fast LP heuristic approach found a solution that was within $0.05 \mathrm{MW}$ of the MILP approach; note that the MILP approach had an optimality gap of $0.01 \%$. For those 33 cases, the fast LP approach converges to a sub-optimal solution. 31 of those cases belonged to the results shown in Fig. 6 and the other 2 belonged to Fig. 8 . These two figures represent placement of FACTS based on the reactance of the lines. The results confirmed that placement of the FACTS devices based on the reactance of the lines is not the best strategy and only under such placement policy did the algorithm converge to suboptimal solutions. For these 33 cases, the maximum difference between the violation reductions obtained by LP and MILP methods were only $0.76 \mathrm{MW}$ and the average difference was just $0.25 \mathrm{MW}$. Therefore, even for the cases that our algorithm was not able to find the optimal solution, it was able to find a solution very close to the optimal solution, much faster than the original MILP problem.

3. Fig. 5, 7, 9, and 11 show that the LP-based method developed in this paper was much faster that the original MILP based formulation. Although the computational time for MILP is not terribly long for the system that is studied in this paper, the results also showed that the MILP computational time was sensitive to the number of FACTS devices and their capacities. Furthermore, for larger systems, the MILP formulation will not scale well and, thus, the computational improvement of the fast LP approach will be even more profound since the LP approach will scale well. The computational results for the LP based formulation were much more consistent across the different cases, meaning that it has a much more robust behavior. Table I summarizes the computational time statistics for the two formulations. Note that these statistics are presented on a single contingency basis, while the information in Fig. 4-7 reflected all contingencies in the entire day.

TABLE I

COMPUTATIONAL TIME STATISTICS FOR LP AND MILP (MS)

\begin{tabular}{l|rrrr}
\hline & \multicolumn{1}{|c}{ Max } & Min & Average & St. Dev. \\
\hline LP & 382 & 8 & 23.4 & 9.2 \\
MILP & 4635 & 36 & 406.5 & 539.0 \\
\hline
\end{tabular}

\section{CONCLUSIONS}

Due to the complexities of modeling N-1 reliability constraints in the day-ahead market problem, reserve requirements are used as a proxy. However, reserve deliverability is not guaranteed, i.e., there is no guarantee that reserve will be deliverable to the needed location. The flexibility of the transmission network can be harnessed to improve the deliverability of the reserve and reduce the need for costly OMC actions. FACTS devices are one particular source of such flexibility. This paper contributes to the literature by reformulating the NLP problem representing optimal corrective adjustment of FACTS set point to an MILP. Engineering insight was then introduced to reduce the computational complexity of the problem to an LP. Although there is no guarantee that the LP formulation converges to the globally optimal solution, the simulation studies showed that the proposed LP-based method was able to find the optimal solution in $98.8 \%$ of the time. Even when optimality was not achieved, the solution was very close to the optimal solution and the computational time was significantly reduced. The proposed method was roughly 17 times faster and showed more robust behavior with respect to the computational time in comparison to the original MILP formulation. Moreover, LPs have shown to have much better scalability properties compared to MILPs, which would translate into even more significant computational time improvements for realistic large-scale power systems. The presented LP-based method was able to find the optimal solution for all the simulations under the FACTS placement policy of installation on more heavily utilized lines, which achieved the best FACTS placement savings.

\section{REFERENCES}

[1] Y. M. Al-Abdullah, M. Abdi-Khorsand, and K. W. Hedman, "The role of out-of-market corrections in day-ahead scheduling," IEEE Trans. Power Syst., vol. 30, no. 4, pp. 1937-1946, July 2015.

[2] G. T. Heydt, R. Ayyanar, K. Hedman, and V. Vittal, "Electric power and energy engineering: the first century," Proceedings of IEEE, vol. 100, no. 2, pp. 1315-1328, May 2012.

[3] E. B. Fisher, R. P. O'Neill, and M. C. Ferris, "Optimal transmission switching," IEEE Trans. Power Syst., vol. 23, no. 3, pp. 1346-1355, Aug. 2008.

[4] K. W. Hedman, R. P. O'Neill, E. B. Fisher, and S. S. Oren, "Optimal transmission switching-sensitivity analysis and extensions," IEEE Trans. Power Syst., vol. 23, no. 3, pp. 1469-1479, Aug. 2008.

[5] K. W. Hedman, R. P. O’Neill, E. B. Fisher, and S. S. Oren, "Optimal transmission switching with contingency analysis," IEEE Trans. Power Syst., vol. 24, no. 3, pp. 1577-1586, Aug. 2009.

[6] K. W. Hedman, M. C. Ferris, R. P. O’Neill, E. B Fisher, and S. S. Oren, "Co-optimization of generation unit commitment and transmission switching with N-1 reliability," IEEE Trans. Power Syst., vol. 25, no. 2, pp. 1052-1063, May 2010.

[7] C. Barrows and S. Blumsack, "Transmission switching in the RTS-96 test system," IEEE Trans. Power Syst., vol. 276, no. 2, pp. 1134-1135, May 2012

[8] A. S. Korad and K. W. Hedman, "Robust corrective topology control for system reliability," IEEE Trans. Power Syst., vol. 28, no. 4, pp. 4042 4051, Nov. 2013.

[9] P. Balasubramanian and K. W. Hedman, "Real-time corrective switching in response to simultaneous contingencies," J. Energy Eng., vol. 141, no. 1, pp. 1-10, 2015.

[10] A. S. Korad, P. Balasubramanian, and K. W. Hedman, "Robust corrective topology control," Handbook of Clean Energy Systems, John Wiley and Sons, pp. 1-17, July 2015.

[11] C. Lehmkoster, "Security constrained optimal power flow for an economical operation of FACTS devices in liberalized energy markets," IEEE Trans. Power Del., vol. 17, no. 2, pp. 603-608, Apr. 2002.

[12] G. N. Taranto, L. M. V. G. Pinto, and M. V. F. Pereira, "Representation of FACTS devices in power system economic dispatch," IEEE Trans. Power Syst., vol. 7, no. 2, pp. 572-576, May 1992.

[13] Y. Xiao, Y. H. Song, C. C. Liu, and Y. Z. Sun, "Available transfer capability enhancement using FACTS devices," IEEE Trans. Power Syst., vol. 18, no. 1, pp. 305-312, Feb. 2003.

[14] H. Mori and Y. Maeda, "Application of two-layered tabu search to optimal allocation of UPFC for maximizing transmission capability," IEEE Intl. Symp. Circ. Syst., 2006.

[15] T. Orfanogianni and R. Bacher, "Steady-state optimization in power systems with series FACTS devices," IEEE Trans. Power Syst., vol. 18 no. 1, pp. 19-26, Feb. 2003.

[16] C. C. Liu, G. T. Heydt, and A. A. Edris, "Impact of FACTS controllers on transfer capability of power grids," IEEE PES Winter Meeting, 2002. 
[17] N. Yorino, E. E. El-Araby, H. Sasaki, and S. Harada, "A new formulation for FACTS allocation for security enhancement against voltage collapse," IEEE Trans. Power Syst., vol. 18, no. 1, pp. 3-10, Feb. 2003.

[18] R. S. Wibowo, N. Yorino, M. Eghbal, and Y. Zoka, "FACTS devices allocation with control coordination considering congestion relief and voltage stability," IEEE Trans. Power Syst., vol. 26, no. 4, pp. 23022310, Nov. 2011.

[19] P. Fairley, "Flexible AC transmission: the FACTS machine," IEEE Spectrum Magazine, Jan. 2011.

[20] G. Hug, "Coordinated power flow control to enhance steady state security in power systems," Ph.D. Dissertation, Swiss Federal Institute of Technology, Zurich, 2008.

[21] M. Amin, "North American electricity infrastructure: system security, quality, reliability, availability, and efficiency challenges and their societal impacts," Continuing Crises in National Transmission Infrastructure: Impacts and Options for Modernization. National Science Foundation (NSF), 2004

[22] M. Sahraei-Ardakani, A. Korad, K. W. Hedman, P. Lipka, and S. Oren, "Performance of AC and DC based transmission switching heuristics on a large-scale polish system," in Proc. IEEE PES General Meeting, July 2014.

[23] M. Sahraei-Ardakani and S. Blumsack, "Market equilibrium for dispatchable transmission using FACT devices," in Proc. IEEE PES General Meeting, July 2012.

[24] M. Sahraei-Ardakani and K. W. Hedman, "A fast LP Approach for enhanced utilization of variable impedance based FACTS devices," IEEE Trans. Power Syst., accepted for publication, 2015.

[25] N. G. Hingorani and L. Gyugyi, Understanding FACTS: concepts and technology of flexible AC transmission systems, Wiley-IEEE Press, Dec. 1999.

[26] ARPA-E GENI, [Online]. Available: http://www.arpae.energy.gov/?q=arpa-e-programs/geni.

[27] J. Mohammadi, G. Hug, and S. Kar, “A Benders' decomposition approach to corrective security constrained OPF with power flow control devices," in Proc. IEEE PES General Meeting, July 2013.

[28] G. Hug, "Generation cost and system risk trade-off with corrective power flow control," in Proc. 50 ${ }^{\text {th }}$ Annu. Allerton Conf., pp. 1324-1333, Oct. 2012.

[29] J. Mohammadi, G. Hug, and S. Kar, "On the behavior of responsive loads in the presence of DFACTS devices," in Proc. North American Power Symp., Sept. 2012.

[30] R. P. O'Neill, E. B. Fisher, B. F. Hobbs, and R. Baldick, "Towards a complete real-time electricity market design," J. Reg. Econ., vol. 34, pp. 220-250, May 2008.

[31] M. Sahraei-Ardakani and S. Blumsack, "Marginal value of FACTS devices in transmission-constrained electricity markets," in Proc. IEEE PES General Meeting, July 2013.

[32] M. Sahraei-Ardakani, "Policy analysis in transmission-constrained electricity markets," Ph.D. Dissertation, Department of Energy and Mineral Engineering, The Pennsylvania State University, University Park, 2013.

[33] M. Sahraei-Ardakani, S. Blumsack, "Transfer capability improvement through market-based operation of series FACTS devices," IEEE Trans. Power Syst., under review, 2015.

[34] Alberto Berizzi, Maurizio Delfanti, Paolo Marannino, Marco Savino and Andrea Silvestri, "Enhanced security constrained OPF with FACTS de- vices," IEEE Trans. Power Syst., vo1.20, no.3, pp. 1597-1605, Aug.2005.

[35] A. Nasiri, A. Conejo, J. Kazempour, M. Ghandhari, "Minimizing wind power spillage using an OPF with FACTS devices," IEEE Trans. Power Syst., vol. 29, no. 5, pp. 2150-2159, Sept. 2014

[36] R. Zárate-Miñano, A. J. Conejo, and F. Milano, "OPF-Based security redispatching including FACTS devices," IET Trans. Gen. Transm. Distrib., vol. 2, no. 6, pp. 821-833, 2008

[37] R. Yang, G. Hug, "Potential and efficient computation of corrective power flow control in cost vs risk trade-off," IEEE Tran. Smart Grid, vol. 5, no. 4, pp. 2033-2043, July 2014.

[38] R. Yang and G. Hug, "Regression-based control of thyristor-controlled series compensators for optimal usage of transmission capacity," IET Gener. Transm. Distrib., vol. 8, no. 8, pp. 1444-1452, Aug. 2014.

[39] W. Shao and V. Vittal, "LP-based OPF for corrective facts control to relieve overloads and voltage violations," IEEE Trans. Power Syst., vol. 21, no. 4, pp. 1832-1839, Nov. 2006.

[40] Dept. Electr. Eng., Univ. Washington, "Power system test case archive," 2007 [Online]. Available: http://www.ee.washington.edu/research/.

[41] S. A. Blumsack, "Network topologies and transmission investment, under electric-industry restructuring," Ph.D. dissertation, Eng. Public Pol., Carnegie Mellon Univ., Pittsburgh, PA, 2006.

[42] M. Sahraei-Ardakani, "IEEE 118-bus test System," [Online]. Available: http://www.public.asu.edu/ msahraei/ieee118.

[43] PJM, "PJM switching solutions,] [Online]. Available: http://www.pjm.com/markets-and-operations/etools/oasis/systeminformation/switching-solutions.aspx.

[44] M. Sahraei-Ardakani, X. Li, P. Balasubramanian, K. Hedman, M. AbdiKhorsand, "Real-time contingency analysis with transmission switching on real power system data," IEEE Trans. Power Syst., accepted for publication, 2015.

Mostafa Sahraei-Ardakani (M'06) received the PhD degree in energy engineering from The Pennsylvania State University, University Park, PA in 2013. He also holds the B.S. and M.S. degrees in electrical engineering from University of Tehran, Iran. Currently, he is a post-doctoral scholar in the School Electrical, Computer, and Energy Engineering at Arizona State University. His research interests include energy economics and policy, electricity markets, power system optimization, transmission network, and the smart grid.

Kory W. Hedman (S' 05, M' 10) received the B.S. degree in electrical engineering and the B.S. degree in economics from the University of Washington, Seattle, in 2004 and the M.S. degree in economics and the M.S. degree in electrical engineering from Iowa State University, Ames, in 2006 and 2007 , respectively. He received the M.S. and Ph.D. degrees in industrial engineering and operations research from the University of California, Berkeley in 2008 and 2010 respectively.

Currently, he is an assistant professor in the School of Electrical, Computer, and Energy Engineering at Arizona State University. He previously worked for the California ISO (CAISO), Folsom, CA, on transmission planning and he has worked with the Federal Energy Regulatory Commission (FERC), Washington, DC, on transmission switching. His research interests include power systems operations and planning, electricity markets, power systems economics, renewable energy, and operations research. 